\title{
The HSBQ Algorithm with Triple-play Services for Broadband Hybrid Satellite Constellation Communication System
}

\author{
Anupon Boriboon and Siriwhaddhanah Pongpadpinit
}

\begin{abstract}
The HSBQ algorithm is the one of active queue management algorithms, which orders to avoid high packet loss rates and control stable stream queue. That is the problem of calculation of the drop probability for both queue length stability and bandwidth fairness. This paper proposes the HSBQ, which drop the packets before the queues overflow at the gateways, so that the end nodes can respond to the congestion before queue overflow. This algorithm uses the change of the average queue length to adjust the amount by which the mark (or drop) probability is changed. Moreover it adjusts the queue weight, which is used to estimate the average queue length, based on the rate. The results show that HSBQ algorithm could maintain control stable stream queue better than group of congestion metric without flow information algorithm as the rate of hybrid satellite network changing dramatically, as well as the presented empiric evidences demonstrate that the use of HSBQ algorithm offers a better quality of service than the traditionally queue control mechanisms used in hybrid satellite network.
\end{abstract}

Keywords-Active Queue Management (AQM), Broadband Hybrid Satellite Constellation Communication System (BHSCCS), COMMStellation $^{\mathrm{TM}}$, Hybrid Satellite - Blue Queue (HSBQ), Queue.

\section{INTRODUCTION}

In the next-generation Global Area Network (GAN), satellite networks are expected to complement the coverage of the globalized networks in instances where they best serve the communication needs of users. In recent years, Micro Systems Canada Inc. (MSCI) [1] has been deployed to provide firm global coverage communications to the public. To increase the feasibility of success of commercial high-speed broadband data satellite networks, it is important for designers to consider both wired and wireless techniques integration together. The initiative of the COMMStellation ${ }^{\mathrm{TM}}$ by MSCI is to supply, produce, deploy, and perform the COMMStellation ${ }^{\mathrm{TM}}$ system on satellite constellation topology over Low Earth Orbit (LEO). The concept provides global backhaul and connects rural areas. Each COMMStellation $^{\mathrm{TM}}$ [2] satellite will provide high

Manuscript received March 16, 2015, revised July 2, 2016.

Anupon Boriboon is with the Vincent Mary School of Science and Technology, Assumption University, Bangkok, Thailand (corresponding author to provide e-mail: anupon_b@hotmail.com).

Siriwhaddhanah Pongpadpinit is with the Department of Business Information System, Martin de Tours School of Management and Economics, Assumption University, Bangkok, Thailand (e-mail: winyu@ @ scitech.au.edu). doi: 10.11601/ijates.v5i2.169 speed, high bandwidth communications to four individual user service provider terminals, with each satellite supporting a total data throughput of up to $8.8 \mathrm{Gbps}(1.1$ Gbps per user service, both uplink and downlink) over 1.6 $\mathrm{GHz}$ of bandwidth. For the ground side of the link both types are Trunk Stations and User Stations. Trunk Stations that always connect to fiber infrastructure will transfer data Internet service with mission concept is bent-pipe configuration. User Stations aims to be a new highbandwidth Internet Service Providers (ISPs), allowing individual clients to connect to its satellite link on the globe. The COMMStellation ${ }^{\mathrm{TM}}$ is designed to provide longhaul wireless broadband global area network communication in an area where optical fiber networks does not exist and large and/or island geographic areas [3].

The Internet wide application which is working with the rapid development of the satellite technique, the hybrid satellite network has been becoming a research focus. For the challenges, efficient utilization of the capital investment in the face of high uncertainty in traffic demand needs to be considered during the hybrid satellite network design stage. A queue sizes oscillations large range make the queue suffer, which will make early congestion notification hard and finally lead to congestion [4].

Recent research activities have placed much emphasis on various the queue weight techniques for the active queue management over satellite segment assuming bottleneck link are known. A variety of Hybrid Satellite - Blue Queue (HSBQ) algorithm aims to evaluate for triple-play service applications over Broadband Hybrid Satellite Constellation Communication System (BHSCCS) [5] using the network system based on COMMStellation ${ }^{\mathrm{TM}}$ satellite system has been proposed in this research. This paper proposes a ratebased stabilization for HSBQ. This algorithm adjusts the queue weight, which is used to estimate the average queue length, based on the rate; moreover, it uses the changes of the average queue length to adjust the amount by the mark or drop of probability which is changed.

This paper is organized as follow: Section II describes the related works which is queue system, Section III is the proposed HSBQ algorithm model, the network topology system module in NS-2 is described in Section IV, Simulation results is presented in Section V, whereas our conclusions are drawn in Section VI. Simulation results show that this algorithm could maintain higher throughput 
than BLUE algorithm as the rate of hybrid satellite network system.

\section{RELATED WORKS}

In this section, we summarize related works. Queues represent locations where packets may be held (or dropped). Packet scheduling refers to the decision process used to choose which packets should be serviced or dropped. Buffer management refers to any particular discipline used to regulate the occupancy of a particular queue. In the common case where a delay element is downstream from a queue, the queue may be blocked until it is re-enabled by its downstream neighbor. This is the mechanism by which transmission delay is simulated. In addition, queues may be forcibly blocked or unblocked at arbitrary times by their neighbors (which is used to implement multi-queue aggregate queues with inter-queue flow control). Packet drops are implemented in such a way that queues contain a drop destination; that is, an object that receives all packets dropped by a queue. This can be useful to keep statistics on dropped packets [6], [7].

In the past few years numerous AQM algorithms have been developed and improved for solving the Internet congestion that appears in routers. The issues that should be considered in AQMs are various problems such as lockout, global synchronization, and fairness. These AQMs used a concept mixture to solve all of the problems above. In analysis AQM schemes were classified to perform the analysis of AQMs with ease. Based on the classification, in general most of the AQMs employed only congestion metric to detect the congestion. However some of the AQMs required additional flow information other than the congestion metric to know the accurate status of the queue. Few of the AQMs required only spotting out the flow information. Considering these AQMs relevant to classification, the first category AQMs based only on congestion metric without flow information such as RED, DS-RED, LRED, HRED, Yellow, REM, BLUE were more simple and easy to design compared to the second category AQMs based on congestion metric with flow information. Therefore, the second category AQMs such as FRED, CHOKe, FAB, SFB also required extra overhead and implementation compared to the first category AQMs. The third category AQMs has a still greater complexity such as SRED, GREEN in identifying the flow information for calculating the marking probability [8].

\section{Proposed the Hybrid SATEllite - Blue Queue ALGORITHM}

The proposal of AQM algorithm is to maintain high link utilization with low loss late and queuing delay in a wide range of network environments. Stable operation and quick response to changes in load are also desirable. This section outlines a proposed algorithm and explains its design in terms of these attributes. We begin with a general discussion of how the proposed algorithm provide control of queuing delay, then provides description of the adjustment of algorithm adapting to changes.

BLUE [9] uses packet loss and links idle events to manage congestion to give a very high throughput and high utilization with low queue length stability. For the better quality of high link utilization and throughput, it should be accessible by Load-based AQMs instead of Queue-based AQMs. Based on the research it indicates that the additional flow information is unnecessary to give attention. In the routers, the congestion is brining awareness get a better strength by Load-based AQMs. During, the comparison of congestion indicated the design of Queue-based AQMs is simple but some except for the parameter tunes for suitable of each problem compared with the other AQMs. In term of satellite high throughput and utilization, the Load-based AQMs performed better than Queue-based by flow information in AQMs.

In AQM schemes, comparative is based on performance metrics of [8], it appears as moderate of loss rate and low queue stability for the BLUE queue. Hence, we have to modify this point to improve BLUE algorithm to make it better for broadband hybrid satellite network system. Also, loss rates are low, and queue stability is higher. It aids to support more link utilization, and throughputs are very high.

Generally of probability which drop the packets before the queues overflow at the space nodes and/or ground nodes in system. An HSBQ (Hybrid Satellite - Blue Queue) is an active queue management algorithm for broadband hybrid satellite network system, which orders to avoid high packet loss rates and control stable stream queue. By-product from this modification it is reduced queue length. The motivations for removing unfairness while maintaining queue length stability is that when the HSBQ scheme decides to discard packets in order to decrease the input traffic before the queues overflow.

We adopt $Z$ parameter from Yellow queue [10] to modify our proposal, the load factor $Z$ will calculate during each averaging interval. The function of Virtual Available Capacity $\hat{c}$ can be updated based on the queue control function, which following [10] Yellow algorithm.

The smoothing parameter $(\alpha)$ and the desired utilization parameter $(\delta)$ are adopted from AVQ algorithm [11] that means we get a theorem to modify our proposal.

Also, toward the HSBQ proposal it has been modified based on BLUE active queue management algorithm, then

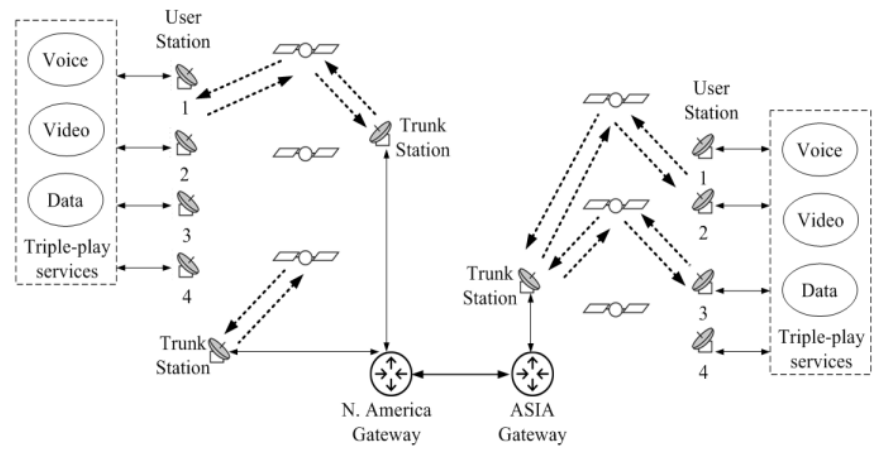

Fig. 1. Broadband hybrid satellite constellation communication system layout. 
we have combination with smoothing parameter, desired utilization parameter, and load factor. HSBQ maintains a single probability $P_{m}$ to mark or drop packets, which the buffer overflows HSBQ algorithm increase $P_{m}$ to increase the congestion notification and decrease to reduce the congestion notification rate in case of buffer emptiness. Hence, a pseudocode of HSBQ is

Upon packet loss $\left(Q_{\text {len }}>L\right)$ event:

if $(($ ( ( currently - recent_update $) \times \beta \times \delta \times \alpha) / \mathrm{C})>$ hold_time $)$

$$
\begin{aligned}
& P_{m=} P_{m}+\delta_{l} \times Z \\
& \text { recent_update }=\text { currently }
\end{aligned}
$$

Upon link idle event:

if $(($ ( ( currently - recent_update $) \times \beta \times \delta \times \alpha) / \mathrm{C})>$ hold_time $)$

$$
\begin{aligned}
& P_{m=} P_{m}-\delta_{2} / Z \\
& \text { recent_update }=\text { currently }
\end{aligned}
$$

Constant:

hold_time : minimum time period between two consecutive update of $p_{m}$

$P_{m}:$ to either mark or drop the packets

$\delta_{l}:$ incremented

$\delta_{2}$ : decremented

$\beta$ : buffer size

$\delta:$ desired utilization of the link

$\alpha$ : smoothing parameter

$\mathrm{C}$ : capacity of link

$Z$ : load factor

\section{IMPLEMENT WITH BROADBAND HYBRID SATELLITE NETWORKS}

In hybrid satellite communication networks, different restrictions and requirements on different links have to be taken into account. Thus, we have selected a typical representative of LEO walker-type satellite constellations with global area network, the space section is COMMStellation $^{\mathrm{TM}}$ satellite constellation [1], [2], [3], [5] composed of 72 operational satellites plus spares divided into 6 polar orbital planes at an altitude of $1000 \mathrm{~km}$ and inclination angle of $90^{\circ}$. The ground section of the link has two types of stations: i) Trunk Stations are 35 based on ground for connected to the high-speed Internet backbone and ii) User Stations. A given User Station is planned to be current or new Internet Service Provider (ISP), which allows individual clients to connect to its satellite link. The satellite constellation parameters are shown in [3], [5]. The satellite system can be linked with the high-speed Internet and other networks by using suitable gateway.

Figure 1 shows the network architecture of the broadband hybrid satellite constellation communication system (BHSCCS) [5] network with two segments: space, ground. We have two gateways connected through a North America and Asia link the bottleneck link. Each User Station pair sends triple-play services packets to destinations. In the Figure 1 illustrates the BHSCCS topology which uses in this work. The RED, BLUE, DropTail, and proposed HSBQ queuing are applied on the queuing system.

In our experiments, the source four User Station links in different areas around South East Asia are chosen to observe traffic network. The source nodes are located in Bangkok-Thailand, Yangon-Myanmar, Vientiane-Laos, and Oil platform-Indian Ocean. The received four nodes are in North America. The destination nodes are at BostonMassachusetts, Tallahassee-Florida, Saint Paul-Minnesota, and Lincoln-Nebraska respectively. Moreover, we assume to decrease link in satellite links bandwidth to be 90.112 Mbps and 11.264 Mbps. For link capacity of Trunk Station is $6.22 \mathrm{Mbps}$ with $1 \mathrm{msec}$. Inside of internet backbone which is used in this work to be the bottleneck link, we assume that each continent has fiber link to connect together and with low latency delay between them. We adapted to $1.55 \mathrm{Mbps}$ with $5 \mathrm{msec}$. The bottleneck link queue operates in bytes mode with buffer size of $400 \mathrm{~KB}$. In addition, we assign error model at $2 \%$. We use bottleneck queue size as an indication of queuing delay in bottleneck.

In this research, new and efficient of active queue management for constellation satellite system mechanisms are proposed into BHSCCS over LEO orbital in network simulation.

For traffic loads, we evaluate the scheme with more realistic traffic. To generate traffic loads in this simulation is [12] triple-play encompasses the provisioning of three services; high-speed Internet, television (Video on Demand or broadcasting video), and Voice over IP through the use of a single broadband connection. Key factors in the tripleplay services delivery constitute the technological advances deriving from the development of appropriate equipment such as:

VOICE: [13], [14] Voice over Internet Protocol (VoIP) is a general term for describing transmission technologies for voice delivery over IP networks. Thus, we choose [15] a perceptual quality model for adaptive VoIP technique to run under our scenario. G.711 PCM codec is suitable for transported voice via the broadband Internet. Therefore we adopt voice traffic techniques for realistic form [15].

VIDEO: [16] Internet Protocol Television (IPTV) is a technology for delivering digital television service over IP networks. We collect up [17] video coding which H.264 scalable video coding (H.264/SVC) for video streams produced IPTV traffic model. This codec is more efficient bandwidth to enhance the quality of video delivered on the Internet.

For video traffic, we adopt the video file foreman with Siemens logo. It is contains 30 frames per second. The length of video is about 30 seconds, so we repeat this video three times into one video file and convert to high

TABLE I

SIMULATIONS TRAFFIC OF TRIPLE-PLAY SERVICES OVER IPV6

\begin{tabular}{cc}
\hline \hline Traffic & Packet size \\
\hline VoIP with G.711 codec & $\sim 220$ bytes \\
IPTV with H.264/SVC & $\sim 1540$ bytes \\
FTP with TCP Westwood & $\sim 540$ bytes \\
\hline \hline
\end{tabular}


definition on $1920 \times 1080$. The summarized traffic of tripleplay services over IPv6 parameters are shown in Table 1.

DATA: [18] Generally, File Transfer Protocol (FTP) is used for handling bulk data transfers. It is used over TCP protocol to communicate, so we adopt the TCP Westwood [19] protocol to run under our scenario. TCP Westwood is designed for efficiency for high speed wireless connection.

\section{Simulation Results}

In coherence to other research, we implement queue management technique to study the most usual type of hybrid satellite constellations based on the BHSCCS network topology within the triple-play services application. We tested the performance of new queue algorithm in terms of drop ratio and average queue length per link. Drop ratio is defined as the ratio of dropped packets to the sum of dropped and successfully transmitted packets, and average queue length is the ratio of the sum in the average number of packets as bottleneck gateways.

The simulations are monitored average queue length, queue bandwidth, and packet end-to-end delay over a period of 100 seconds. The load consists of a combination of VoIP G.711 codec, IPTV H.264/SVC, and FTP over TCP Westwood. The simulation results for different AQMs loadings such as BLUE, RED, DropTail and proposed HSBQ, in which the loss rate ranges at $2 \%$.

Voice and video traffic are different packet sizes. But their performances in each AQMs is affected by packet endto-end delay in transmitted packet rate in same way of a predefined delay threshold. So, Figure 2 (a) DropTail, (b) RED, (c) BLUE, and (d) proposal HSBQ show that packet end-to-end delay of VoIP G.711 with two way connections. We also run simulations for comparison with other AQMs. The graph shows that RED suffers from continual unstable of packet arrival time because RED has random dropped packet in queue due to the deterministic packet marking behavior. Proposed HSBQ is lowest packet end-to-end delay than BLUE and DropTail queues. That means propagation delay of VoIP G.711 with two way connections from source to destination for our proposal over broadband hybrid satellite network has the lowest delays. It has shown the communicated by voice is rather smooth traffic voice because lowest propagation delays in end-to-end communication than DropTail and BLUE.

Figure 3 (a) DropTail, (b) RED, (c) BLUE, and (d) proposed HSBQ shows packet end-to-end delay of IPTV H.264/SVC with $1920 \times 1080$ resolutions at 30 frames per second. We also run simulations for comparison with other AQMs. The graph shows that proposed HSBQ is lowest packet end-to-end delay than BLUE and DropTail queues, but it is higher than RED because RED is random dropped packet in queue behavior. That means propagation delay from source to destination for our proposed over broadband hybrid satellite network has the lowest delays. Proposed HSBQ is lowest packet end-to-end delay than BLUE and DropTail queues. That means propagation delay of IPTV H.264/SVC from source to destination for our proposal over broadband hybrid satellite network has the lowest delays. It has shown the communication by IPTV has a rather smoother traffic voice because of lowest propagation delays in end-to-end communication than DropTail and BLUE.

For Figure 4 we have shown the PSNR results to reflect frame number of video in variations of each AQMs. The

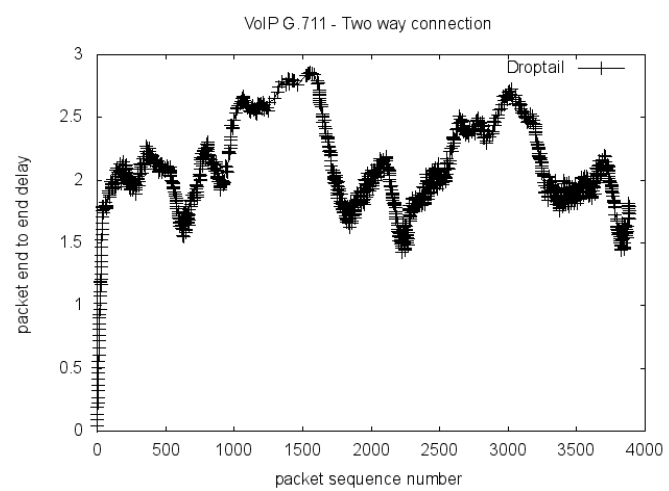

(a)

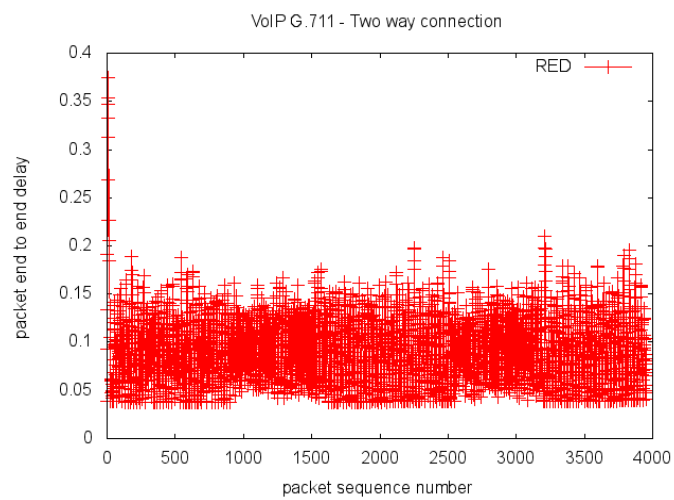

(b)

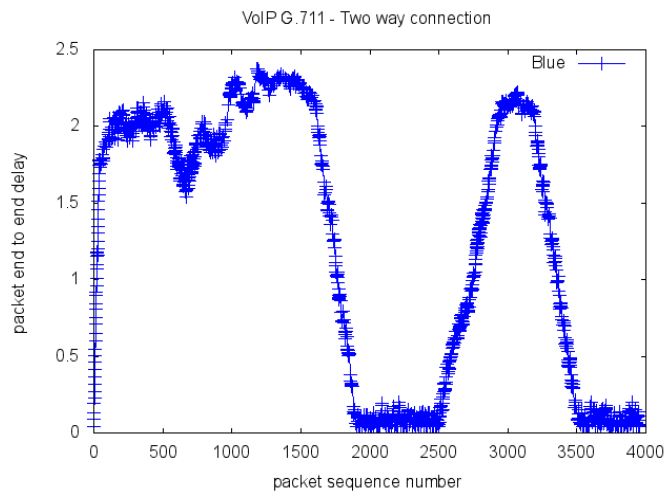

(c)

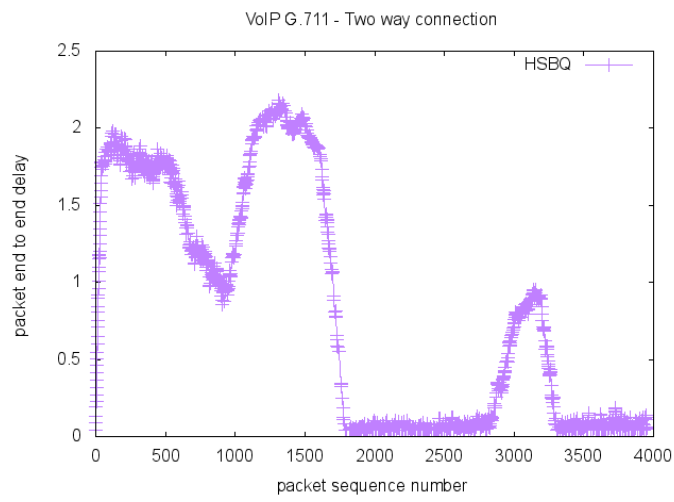

(d)

Fig. 2. VoIP: packet end-to-end delay in different queue (a) DropTail; (b) RED; (c) BLUE; (d) HSBQ 
PSNR is an approximation to human perception of reconstruction image quality, however a higher PSNR generally indicates that the reconstruction is of higher quality but in some cases it may not. Also, the result of PSNR values for IPTV in different AQMs in simulation

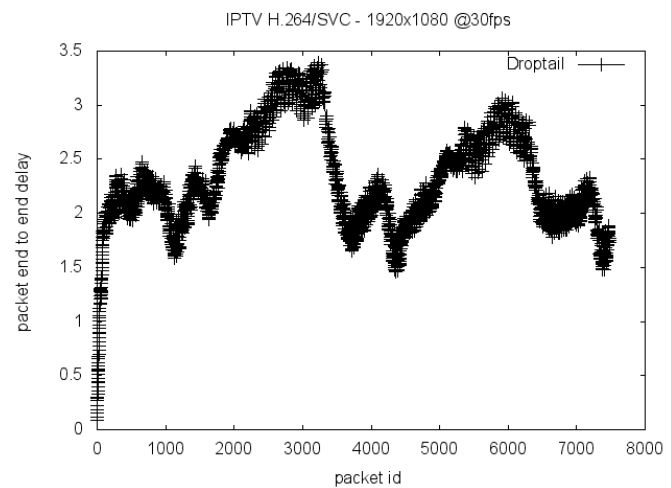

(a)

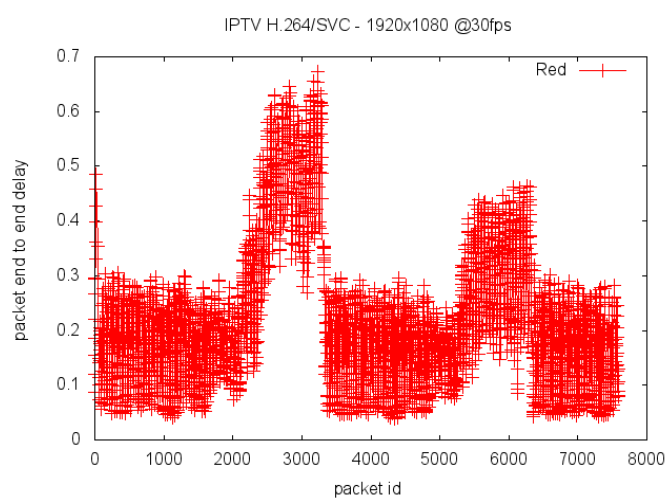

(b)

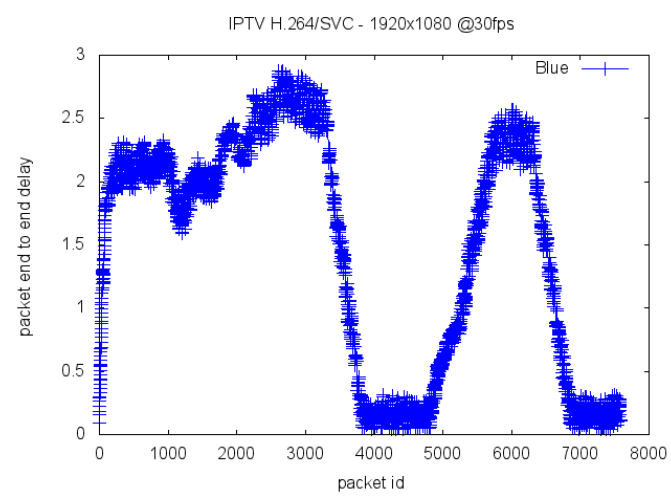

(c)

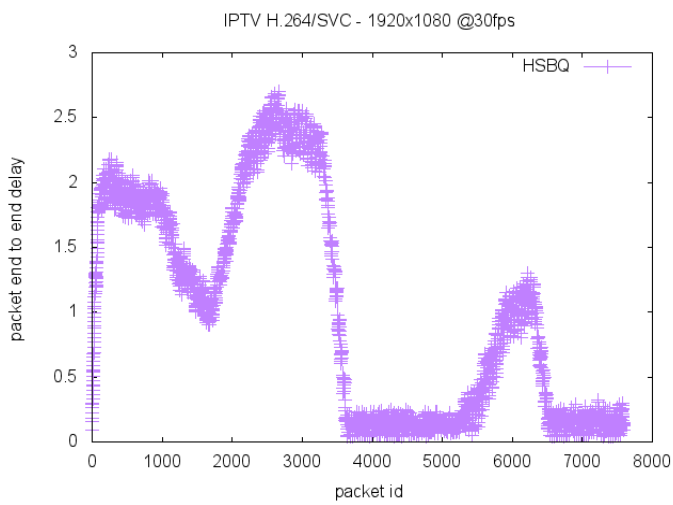

(d)

Fig. 3. IPTV: packet end-to-end delay in different queue (a) DropTail; (b) RED; (c) BLUE; (d) HSBQ with foreman Siemens logo are shown in Figure 4 (a), (b), (c), (d) respectively.

From Figure 4 in axis $\mathrm{x}$ shows frame number of video that was received in destination node. DropTail is lowest frame number of video than other queues in group of

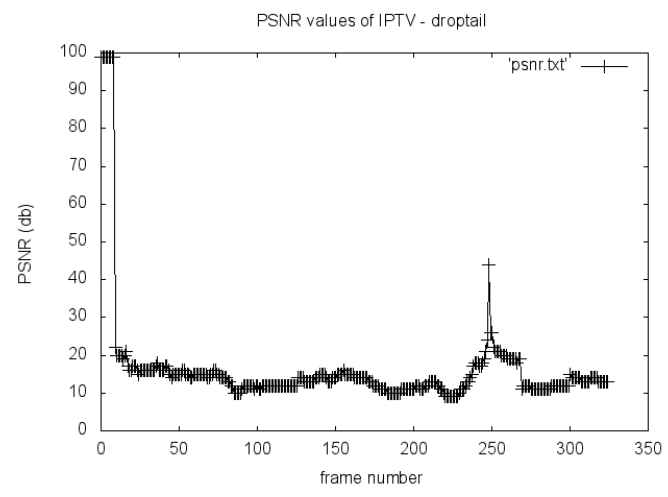

(a)

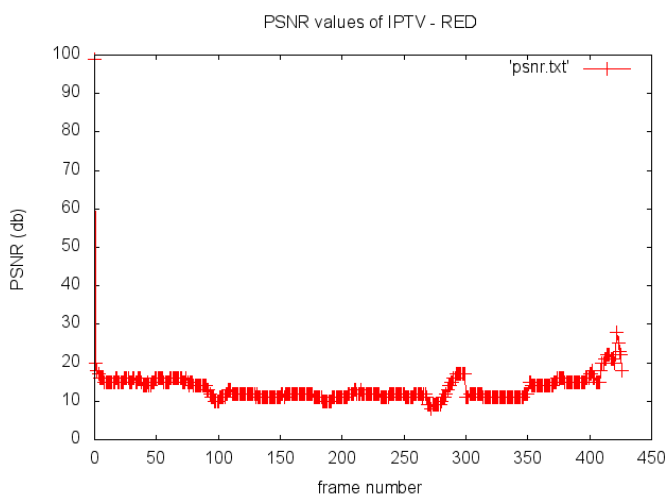

(b)

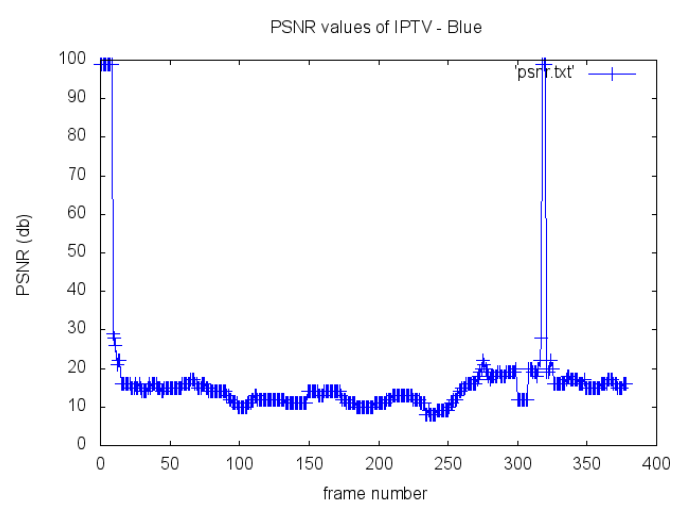

(c)

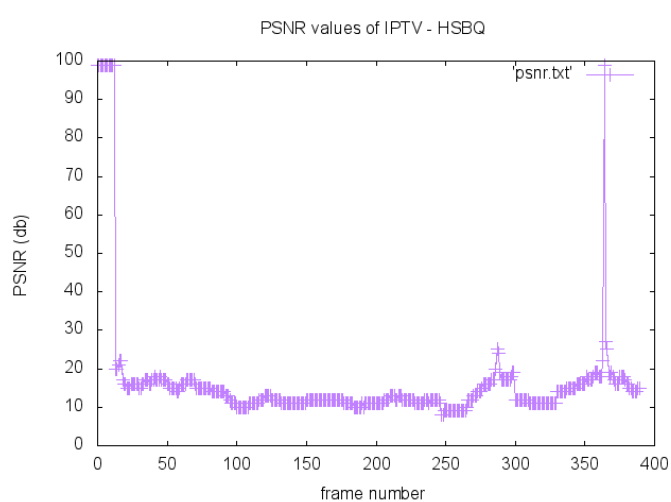

(d)

Fig. 4. PSNR value of IPTV in different queue (a) DropTail; (b) RED; (c) BLUE; (d) HSBQ 
simulation, that mean reconstruction image quality of video is lower. But RED is the highest frame number of videos than other queues in group of simulation, that means highly reconstruction image quality, however a higher of RED that does not prove a smoother video frame that is shown in Figure 5. Next is our proposal HSBQ shown frame number of video is more than DropTail and BLUE. The smoother video frame from reconstruction image quality of HSBQ is show in Figure 5.

As a result, we choose frames number 41 of video traffic to visually compare the performance for each AQMs. Figure 5 (a) is the original frame number 41 of source file at resolutions $1920 \times 1080$ over codec H.264/SVC. Figure 5

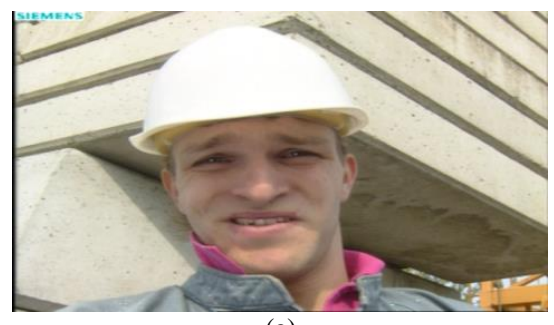

(a)

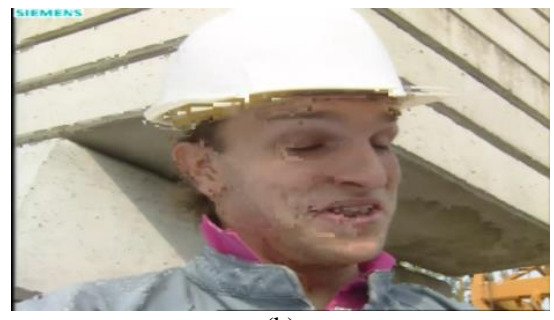

(b)

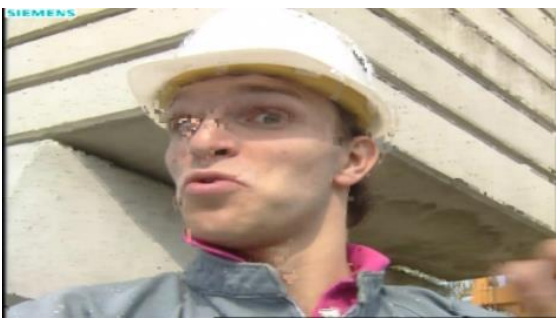

(c)

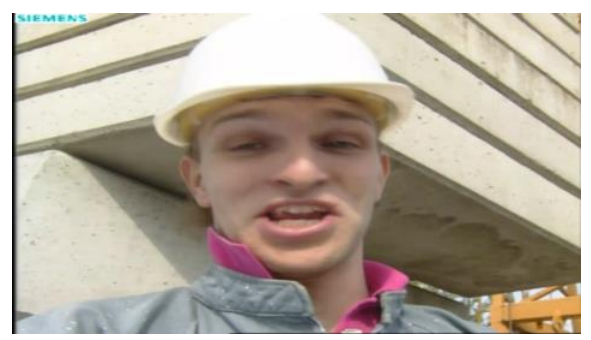

(d)

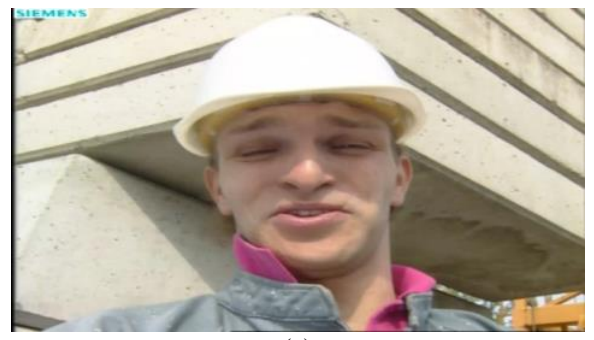

(e)

Fig. 5. Frame no 41 of IPTV in different queue (a) Original; (b) DropTail; (c) RED; (d) BLUE; (e) HSBQ (b), 5 (c), 5 (d), and 5 (e) are DropTail, RED, BLUE, HSBQ respectively. Illustrated shows the smooth motion of the video. In this research, DropTail and RED algorithms are suffering smooth IPTV H.264/SVC over broadband

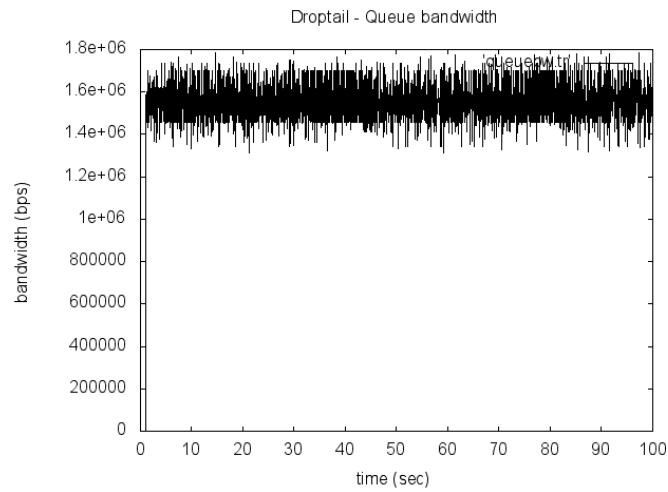

(a)

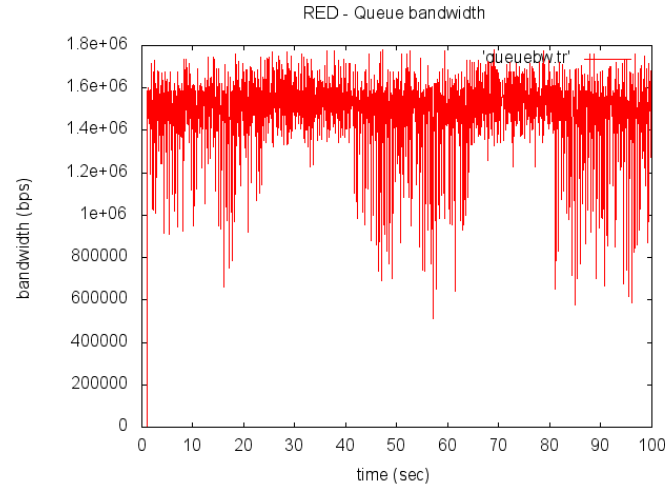

(b)

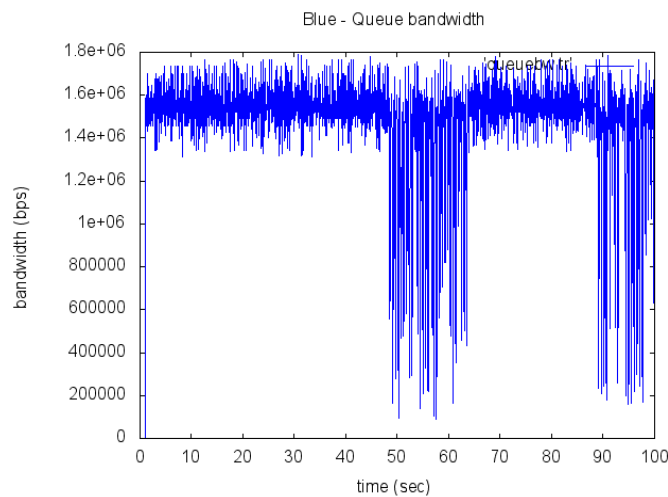

(c)

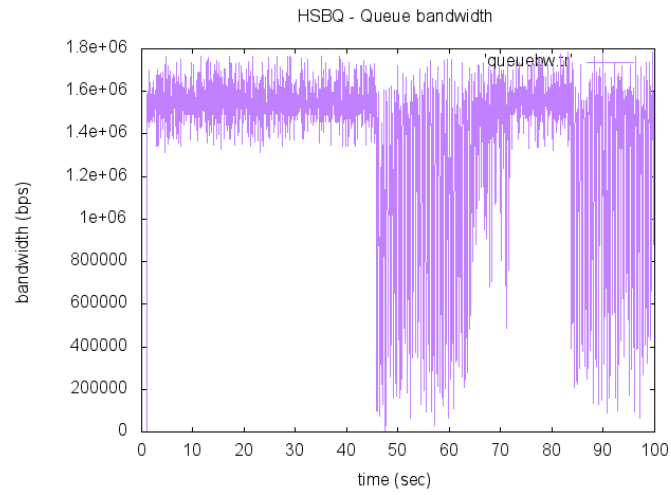

(d)

Fig. 6. Queue bandwidth in different queue (a) DropTail; (b) RED; (c) BLUE; (d) HSBQ 
hybrid satellite communication networks. On the contrary, BLUE and HSBQ algorithms are very smooth. And proposed HSBQ is the lowest dropped packet of video than BLUE, also HSBQ has a frame number more than BLUE.

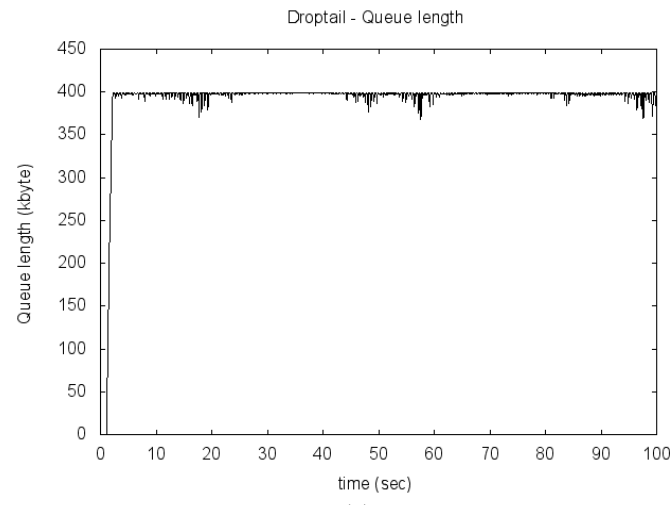

(a)

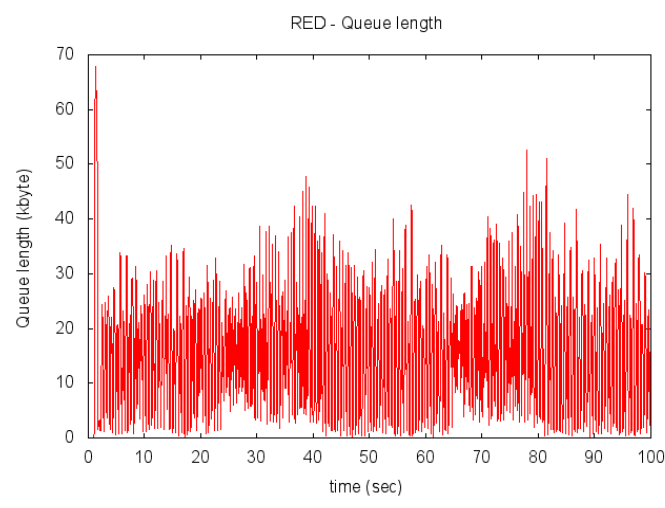

(b)

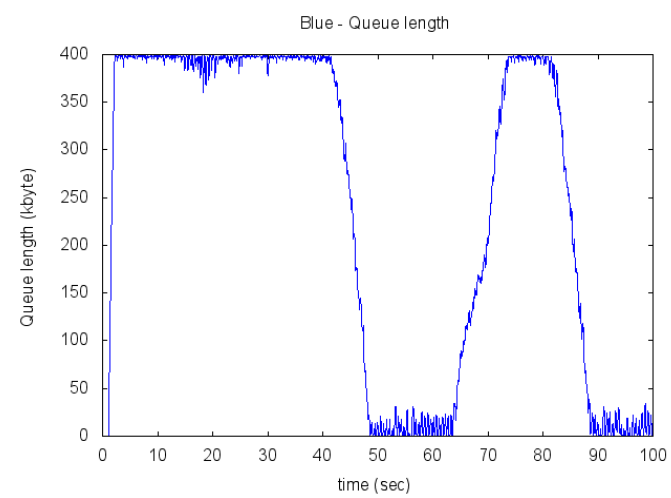

(c)

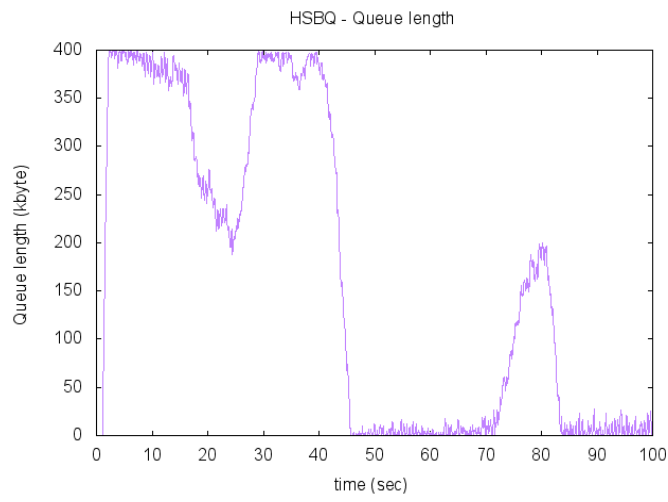

(d)

Fig. 7. Queue length in different queue (a) DropTail; (b) RED; (c) BLUE; (d) HSBQ
In Figure 6 we present the queue bandwidth of triple-play services flows. It is related queue length, and it should be matched up with queue length. These figures show that proposed HSBQ has the lowest uses of queue bandwidth in the traffic networks. That means, queuing in the system are lower than conventional queues. Therefore, traffic in hybrid satellite networks will be free more for other applications in the same time.

In Figure 7 we present the queue length with respect to time. Figure 7 illustrates instantaneous queue length of this simulation with $400 \mathrm{~KB}$ buffer size in triple-play services traffic loads. In proposed HSBQ, queue length decreases to reduce the congestion and reduces oscillatory behaviors. It is clearly shown that the proposed scheme effectively maintains queue length stability around the target queue length whereas the queue length in BLUE oscillates periodically than the proposed HSBQ. Using RED, the queue length is too low, and it is easy for it to be empty, which causes underutilization. Using DropTail is almost full queue length all the time of simulation and hardly increases over $400 \mathrm{~KB}$. They vary the bottleneck queue length affected when varying the simulation parameters in triple-play services traffic loads.

\section{CONCLUSIONS}

Broadband hybrid satellite networks are best suited for covering global area network, density traffic networks, providing a variety of mainly triple-play services application, and are expected to play an important role in the next-generation satellite networks. Numerous AQMs algorithm has been proposed to provide for reducing collision voice, video, data traffic in a hybrid Internet network environment, which is highly dynamic.

In this paper, we have proposed a new queue management scheme. It is the HSBQ algorithm classified based on congestion metrics and without the flow information. The performance of active queue management algorithms was evaluated in terms of drop ratio and average queue length per link. The simulation results have shown that the HSBQ algorithm significantly improves bottleneck network performance in comparison with a conventional AQMs algorithm. The queue bandwidth and queue length of HSBQ are steady while maintaining queue in order to decrease triple-play service application traffic before the queues overflow. The results obtained show that the proposed algorithm is capable of handling varying traffic distributions like triple-play services. However, the proposed algorithm has higher performance improvement and lower packet end-to-end delays in BHSCCS. In addition, we will apply the presented analytical of our model in clustering network system which is important from the network operator perspective.

\section{REFERENCES}

[1] G. J. Wells, and D. Cooper, "COMMStellation Implementations for Northern Broadband Communications," in Proc. 30th AIAA International Communications Satellite System Conference (ICSSC), Canada, 2012, pp. 830-839. 
[2] G. J. Wells, D. Cooper, P. Sekhavat, S. Engleson, and P. Takats, "COMMStellation ${ }^{\mathrm{TM}}$ - A Low Latency Satellite Constellation for Broadband Communications," in Proc. 30th AIAA International Communications Satellite System Conference (ICSSC), Canada, 2012, pp. $858-871$.

[3] A. Boriboon, and S. Pongpadpinit, "The COMMStellation ${ }^{\mathrm{TM}}$ Satellite Constellation for Broadband Communication System Model in NS-2," Int. J. Communications, Network and System Sciences, vol. 7 no. 10, pp. 430-439, Oct. 2014

[4] Y. Ma, T. Zhang, and J. Zhang, "An AQM Algorithm for LEO Satellite Network," in Proc. IEEE 4th International Symposium on Microwave, Antenna, Propagation, and EMC Technologies for Wireless Communications (MAPE), Beijing, 2011, pp. 615-618.

[5] A. Boriboon, and S. Pongpadpinit, "Optimized routing protocol for broadband hybrid satellite constellation communication IP network system," EURASIP Journal on Wireless Communications and Networking, vol. 2016, no. 1, pp. 1-11, May. 2016.

[6] C. Joo, S. Bahk, and S. S. Lumetta, "A Hybrid Active Queue Management for Stability and Fast Adaptation," Journal of Communications and Networks, vol. 8, no. 1, pp. 93-105, March. 2006.

[7] K. Fall, and K. Varadhan (ed.), The ns Manual (formerly ns Notes and Documentaion). UC Berkeley, CA: UC Berkeley, LBL, USC/ISI, and Xerox PARC, 2009.

[8] K. Chitra, and G. Padamavathi, "Classification and Performance of AQM-Based Schemes for Congestion Avoidance," Int. J. of Computer Science and Information Security, vol. 8, no. 1, pp. 331-340, April. 2010.

[9] J. Kim, H. Yoon, and I. Yeom, "Active Queue Management for Flow Fairness and Stable Queue Length," IEEE Trans. on Parallel and Distributed Systems, vol. 22 no. 4, pp. 571-579, April. 2011.

[10] C. Long, B. Zhao, X. Guan, and J. Yang, "The Yellow active queue management algorithm," J. Computer Networks and ISDN Systems, vol. 47, no. 4, pp. 525-550, March. 2005.

[11] S. S. Kunniyur, and R Srikant, "An Adaptive Virtual Queue (AVQ) Algorithm for Active Queue Management," IEEE/ACM Transaction on Networking, vol. 12, no. 2, pp. 286-299, April. 2004. doi:10.1109/TNET.2004.826291

[12] G. P. Sotiropoulos, D. K. Styliaras, E. A. Kosmatos, C. A. Papagianni, N. D. Tselikas, and I. S. Venieris, "Triple Play Service Simulation and Packet Scheduling Performance Evaluation," in Proc. the International Conference on Digital Telecommunications (ICDT '06), France, 2006, pp. 54-59.

[13] M. Ali, L. Liang, Z. Sun, and H. Cruickshank, "Evaluation of Transport Protocols for SIP Signaling over IPv6 DVB-RCS Satellite Networks," in Proc. 7th International Symposium on Wireless Communication Systems (ISWCS), 2010, UK, pp. 800-804.

[14] M. Ali, L. Liang, Z. Sun, and H. Cruickshank, "SIP Signaling and QoS for VoIP over IPv6 DVB-RCS Satellite Networks," in Proc. the International Workshop on Satellite and Space Communications (IWSSC 2009), 2009, Italy, pp. 419-423.

[15] A. Bacioccola, C. Cicconetti, and G. Stea, "User-level performance evaluation of VoIP using ns-2," in Proc. 2nd International Conference on Performance Evaluation Methodologies and Tools (ValueTools '07), 2007, Belgium, pp. 1-10.

[16] S. Pongpadpinit, "Motion vector recovery based colour information," in Proc. 17th International Conference on Digital Signal Processing (DSP), 2011, Greece, pp. 1-4.

[17] C. Ke, "myEvalSVC: an Integrated Simulation Framework for Evaluation of H.264/SVC Transmission," J. KSII Transactions on Internet and Information Systems, vol. 6, no. 1, pp. 379-394, Jan. 2012.

[18] P. Lai, H. Subramoni, S. Narravula, A. Mamidala, and D. K. Panda, "Designing Efficient FTP Mechanisms for High Performance DataTransfer over InfiniBand," in Proc. the International Conference on Parallel Processing (ICPP '09), Vienna, 2009, pp. 156-163.

[19] C. Casetti, M. Gerla, S. Mascolo, M. Y. Sanadidi, and R. Wang, "TCP Westwood: End-to-End Congestion Control for Wired/Wireless Networks," J. Wireless Networks, vol. 8, no. 5, pp. 467-479, Sept. 2002. 